Ciencia y Salud, Vol. V, No. 2, mayo-agosto, 2021 • ISSN (impreso): 2613-8816•ISSN (en línea): 2613-8824

DOI: https://doi.org/10.22206/cysa.2021.v5i2.pp87-94

\title{
DESCRIPCIÓN DEL RIESGO CARDIOVASCULAR EN PACIENTES DIABÉTICOS TIPO 1 EN REPÚBLICA DOMINICANA
}

\author{
Type 1 diabetes cardiovascular risk description \\ in Dominican Republic
}

\author{
Anthony Gutiérrez Martínez ${ }^{\mathrm{a}}$, Moisés Vásquez ${ }^{\mathrm{b}}$, Robin Ferreras ${ }^{\mathrm{c}}$, Ivonne Canto ${ }^{\mathrm{d}}$ \\ y Katherine Calderón ${ }^{\mathrm{e}}$
}

Recibido: 6 de mayo, $2020 \bullet$ Aprobado: 22 de agosto, 2020

Cómo citar: Martínez AG, Vásquez M, Ferreras R, Canto I, Calderón K. Descripción del riesgo cardiovascular en pacientes diabéticos tipo 1 en República Dominicana. cysa [Internet]. 10 de junio de 2021 [citado 16 de junio de 2021];5(2):87-4. Disponible en: https:// revistas.intec.edu.do/index.php/cisa/article/view/2223

\section{Resumen}

Introducción: la diabetes tipo 1 es una enfermedad crónica de alto impacto económico con gran capacidad de ser controlada, la misma no tiene ninguna descripción local previa. Su principal causa de mortalidad es los eventos cardiovasculares y el manejo adecuado la disminuye considerablemente.

Objetivo: determinar el riesgo cardiovascular en pacientes adultos con diabetes tipo 1 en la ciudad de Santiago de los Caballeros, República Dominicana.

Método: se realizó un estudio descriptivo transversal multicéntrico con 39 pacientes en el período de junio a noviembre de 2019. La calculadora "Steno T1 Risk Engine" se utilizó para estimar el riesgo cardiovascular.

Resultados: se obtuvo una relación significativa entre la albuminuria $(\mathrm{p}=0.0127)$, presión arterial sistólica $(\mathrm{p}=$ 0.0002), tiempo de diagnóstico ( $\mathrm{p}=0.0037$ ) y nivel de riesgo cardiovascular. La hemoglobina glucosilada $(\mathrm{p}=0,7884)$ y la

a Pontificia Universidad Católica Madre y Maestra, Facultad Ciencias de la Salud, Escuela de Medicina. Correo-e: anthonyGutierrez@gmail.com, ORCID: 0000-0003-4567-0123

b Pontificia Universidad Católica Madre y Maestra, Facultad Ciencias de la Salud, Escuela de Medicina, Correo-e: 20140600@ce.pucmm.edu.do

\begin{abstract}
Introduction: Type 1 diabetes is a chronic condition with a high economic impact but potentially controllable. There is no previous local description of this condition. Its main fatality cause is due to cardiovascular events and its proper management can diminish it.

Objective: This study aimed to determine the cardiovascular risk in adult patients with type 1 diabetes mellitus in the city of Santiago de los Caballeros, Dominican Republic.

Method: A descriptive multicenter cross-sectional study was done on 39 patients in the period of June-November 2019. "Steno T1 Risk Engine" calculator estimated the cardiovascular risk.

Results: A significant relationship was obtained between albuminuria $(\mathrm{p}=0.0127)$, systolic blood pressure $(\mathrm{p}=0.0002)$, diagnosis time $(\mathrm{p}=0.0037)$ and cardiovascular risk level.

\footnotetext{
c Pontificia Universidad Católica Madre y Maestra, Facultad Ciencias de la Salud, Escuela de Medicina Correo-e: 20140975@ce.pucmm.edu.do de la Salud, Escuela de Medicina.Correo-e: ii.canto@ce.pucmm.edu.do
}

d Pontificia Universidad Católica Madre y Maestra, Facultad Ciencias

e Pontificia Universidad Católica Madre y Maestra, Facultad Ciencias de la Salud, Escuela de Medicina. Correo-e: kncalderon@gmail.com
\end{abstract}


actividad física $(\mathrm{p}=0.706)$ no mostraron una relación significativa con el riesgo cardiovascular.

Conclusión: el nivel de riesgo cardiovascular promedio es bajo, con probabilidades $<10 \%$ de un evento cardiovascular agudo dentro de los 10 ańos. Esta herramienta permite incluir una evaluación cardiovascular rutinaria con datos que perfilen el tratamiento orientado a disminuir complicaciones vasculares, mortalidad y aumentar adherencia al tratamiento.

Palabras clave: diabetes mellitus tipo 1; enfermedades cardiovasculares; Atención Primaria de Salud; República Dominicana; diabetes mellitus.

\section{Introducción}

La diabetes tipo 1 (DT1) es una alteración metabólica caracterizada por niveles altos de glucosa en sangre debido a la falta de secreción de insulina. Hay varios tipos de diabetes con base en la fisiopatología detrás de ella. La diabetes tipo 2, caracterizada por una resistencia progresiva a la insulina, se ha descrito a fondo en contraste con la DT1, que localmente tiene una prevalencia menor ${ }^{1-3}$. Debido a su naturaleza crónica y gravedad, surgen complicaciones como eventos cardiovasculares (CV), siendo la principal causa de mortalidad en la DT1, mostrando un aumento de 10 veces en la fatalidad asociada a riesgos CV con una hemoglobina glucosilada $(\mathrm{HbA} 1 \mathrm{c})>9.7 \%$. El espectro de la diabetes es responsable de 1,5 millones de muertes/año a nivel mundial, siendo actualmente del $11 \%$, en la población de la República Dominicana $(\mathrm{RD})^{3-6}$. Los estudios económicos han encontrado que para el 2015, RD gastó 792 millones de dólares en diabetes en general y las complicaciones CV representaron la mayor carga económica, siendo estas más comunes en la DT1 ${ }^{7}$.

La duración de la diabetes y la edad del paciente son factores de riesgo independientes, reconocidos para
Glycated hemoglobin $(\mathrm{p}=0.7884)$ and physical activity $(\mathrm{p}=0.7063)$ did not show a significant relationship with cardiovascular risk.

Conclusion: Average cardiovascular risk level is low with $<10 \%$ probabilities of an acute cardiovascular event within 10 years. This tool could lead to quick cardiovascular risk evaluations to guide the treatment lowering vascular complications, mortality and increasing treatment adherence

Keywords: Type 1 diabetes; Cardiovascular disease; Primary healthcare; Dominican Republic; Diabetes mellitus.

complicaciones CV y están asociados a la mortalidad en pacientes con DT $1^{8}$. Hasta el $17 \%$ de estos pueden desarrollar eventos cerebrovasculares, miocardiopatía isquémica, enfermedad arterial periférica o insuficiencia cardíaca en un seguimiento a 10 años 9 . El manejo adecuado es importante ya que el seguimiento periódico con un tratamiento intensivo demostró disminuir las complicaciones $\mathrm{CV}$ en la DT $1^{10}$.

En cuanto a las complicaciones, el costo y la mortalidad, se necesitan métodos eficientes de detección de riesgo CV para abordar los perfiles $\mathrm{CV}$ individuales y las posibles complicaciones de cada paciente. Debido a la falta de datos, los factores de riesgo de DT1 asociados con eventos CV no se han evaluado regionalmente. Este estudio tuvo como objetivo determinar el riesgo cardiovascular de los pacientes con DT1 en la República Dominicana.

\section{Métodos}

Este estudio multicéntrico, transversal y descriptivo se centró en pacientes diabéticos tipo 1 que asistieron a consulta de endocrinología en cuatro centros de salud terciarios de Santiago de los Caballeros, RD, 
desde junio a noviembre de 2019. Los datos se obtuvieron del perfil clínico, los resultados de los análisis de sangre y los exámenes físicos realizados por los profesionales de la salud.

Figura 1. Escrutinio de pacientes. Describe el flujo de pacientes desde el reclutamiento hasta el análisis.

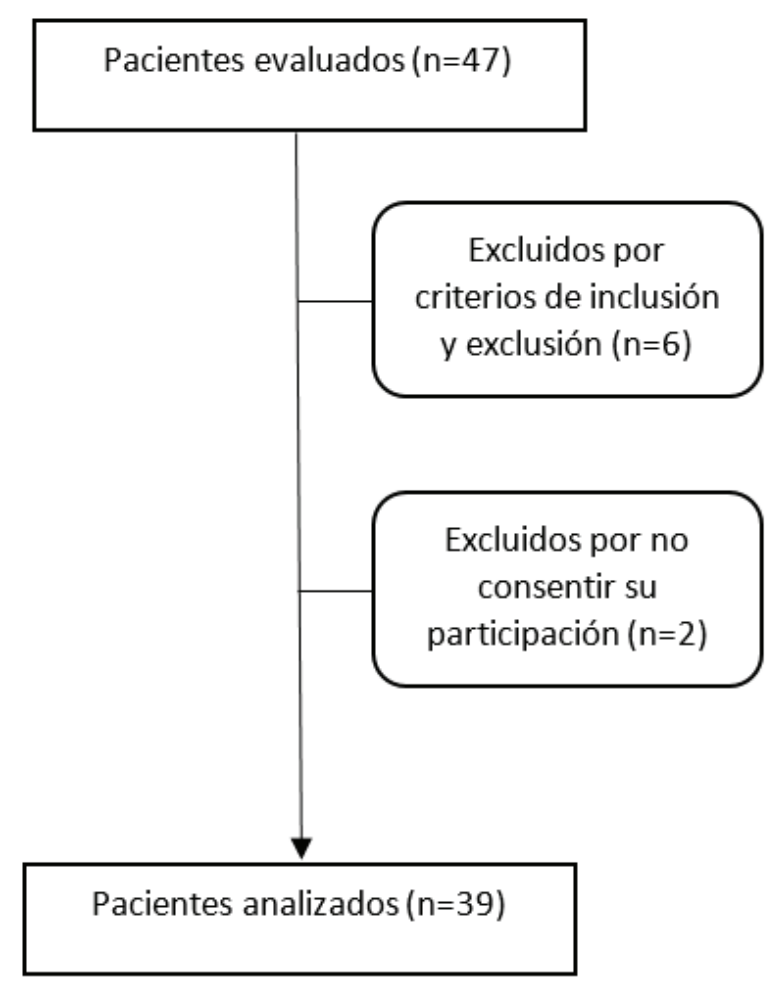

Se incluyeron un total de 39 pacientes con los siguientes criterios de inclusión:

- Diagnóstico y tratamiento previo de DT1 por un profesional de la salud

- Edad entre 18 y 65 años.

- Ser paciente del departamento de endocrinología en uno de los cuatro centros de salud participantes.
- Tener un expediente en uno de los cuatro centros de salud participantes.

- Los criterios de exclusión fueron:

- Participantes con un embarazo en proceso.

- Pacientes con una patología oncológica previa o en curso.

- Participantes admitidos a través de la sala de emergencias.

- Pacientes con un evento cardiovascular previo.

- No consentir a participar en la investigación.

El riesgo de CV a 10 años se calculó a través de la calculadora "Steno T1 Risk Engine" a partir de los datos expuestos en la tabla 1. Se clasifica como riesgo bajo (<10\%), moderado (10-20\%) y grave $(>20 \%)$ de desarrollar un evento agudo de CV en los próximos 10 años ${ }^{11}$. La prueba analítica de chi-cuadrado comparó el riesgo categórico calculado con la actividad física y la albuminuria, one-way ANOVA comparó el tiempo desde el diagnóstico, la HbA1c y los niveles de tensión sistólica. La normalidad de los datos se confirmó con un estudio piloto previo utilizando una evaluación gráfica a través de un histograma, asimetría y valores de curtosis. Las variables continuas se expresan como medias y desviación estándar. La significancia estadística se definió por un valor de $\mathrm{P}<0,05$.

Se eligieron variables objetivas provistas por profesionales de la salud para reducir el sesgo de medición. El tamaño final del estudio se determina mediante reclutamiento continuo desde de junio a noviembre como punto de corte. Los datos faltantes se manejaron considerando casos completos exclusivamente y se obtuvo la autorización previa de un comité de bioética. 


\section{Resultados}

Tabla 1. Descripción clínica y sociodemográfica de la muestra

\begin{tabular}{|c|c|c|}
\hline \multicolumn{2}{|c|}{ Variable } & Media (SD) \\
\hline \multicolumn{2}{|c|}{ Edad (años) } & $27.4( \pm 6.9)$ \\
\hline \multicolumn{2}{|c|}{ Duración de la diabetes (años) } & $14.5( \pm 7.0)$ \\
\hline \multicolumn{2}{|c|}{$\mathrm{LDL}(\mathrm{mg} / \mathrm{dL})$} & $107( \pm 40.0)$ \\
\hline \multicolumn{2}{|c|}{$\mathrm{HbA1c}(\mathrm{mmol} / \mathrm{mol})$} & $74( \pm 2)$ \\
\hline \multicolumn{2}{|c|}{ HbA1c (\%) } & $8.9( \pm 2.3)$ \\
\hline \multicolumn{2}{|c|}{ Presión sanguínea sistólica (mmHg) } & $123( \pm 14.4)$ \\
\hline \multicolumn{2}{|c|}{$\mathrm{eGFR}(\mathrm{ml} / \mathrm{min} / 1.73 \mathrm{~m})$} & $99( \pm 70.7)$ \\
\hline Variable & Categoría & $\mathbf{N}(\%)$ \\
\hline \multirow{2}{*}{ Sexo } & Masculino & $11(28.21 \%)$ \\
\hline & Femenino & $28(71.79 \%)$ \\
\hline \multirow{3}{*}{ Albuminuria } & $\begin{array}{l}\text { Normoalbuminuria }(< \\
30 \mathrm{mg} / \mathrm{g} \mathrm{Cr})\end{array}$ & $13(33.33 \%)$ \\
\hline & $\begin{array}{l}\text { Microalbuminuria (30 } \\
\quad-300 \mathrm{mg} / \mathrm{g} \mathrm{Cr} \text { ) }\end{array}$ & $13(33.33 \%)$ \\
\hline & $\begin{array}{l}\text { Macroalbuminuria } \\
\text { (>300 } \mathrm{mg} / \mathrm{g} \mathrm{Cr})\end{array}$ & $13(33.33 \%)$ \\
\hline \multirow{2}{*}{ Fumador activo } & Sí & $0(0 \%)$ \\
\hline & No & $39(100 \%)$ \\
\hline \multirow{2}{*}{$\begin{array}{l}\text { Actividad física } \\
\text { regular }\end{array}$} & $\begin{array}{c}\text { Sí (>3.5 horas a la } \\
\text { semana) }\end{array}$ & $14(32.43 \%)$ \\
\hline & $\begin{array}{c}\text { No }(<3.5 \text { horas a la } \\
\text { semana })\end{array}$ & $25(67.57 \%)$ \\
\hline \multirow{3}{*}{$\begin{array}{l}\text { Riesgo cardio- } \\
\text { vascular }\end{array}$} & Bajo & $26(66.67 \%)$ \\
\hline & Moderado & $6(15.38 \%)$ \\
\hline & Severo & $7(17.95 \%)$ \\
\hline
\end{tabular}

Fuente: elaboración propia.

Un total de 39 pacientes fueron analizados con media de edad de 27,4 [6,9] años y 28 [79,8\%] pacientes fueron femeninas. El tiempo medio desde el diagnóstico fue de 14,5 (7,0) años. Los pacientes tenían una HbAlc media (\%) de $74 \mathrm{mmol} / \mathrm{mol}$ $(8.9 \%)$ y una presión arterial sistólica media (DE) de 123 (14.4) $\mathrm{mmHg}$.

Un total de $25(67,6 \%)$ pacientes informaron actividad física regular, mientras que ninguno era fumador actual. Las categorías de albuminuria mostraron una distribución similar del 33,3\%. La categoría de riesgo cardiovascular predominante fue de bajo riesgo con $66.7 \%$ (figura 1).
Tabla 2. Descripción de variables por categoría riesgo cardiovascular

\begin{tabular}{|c|c|c|c|c|}
\hline \multirow{2}{*}{ Variable } & \multicolumn{3}{|c|}{ Riesgo cardiovascular } & \multirow{2}{*}{$\begin{array}{l}\text { Valor } \\
\text { de P }\end{array}$} \\
\hline & Leve & Moderado & Severo & \\
\hline $\begin{array}{c}\text { Tiempo } \\
\text { desde el } \\
\text { diagnóstico } \\
\text { (años) }\end{array}$ & $\begin{array}{c}11.8 \\
( \pm 5.7)\end{array}$ & $18( \pm 8.1)$ & $21( \pm 4.6)$ & 0.0037 \\
\hline $\begin{array}{l}\text { Presión } \\
\text { sanguínea } \\
\text { sistólica } \\
(\mathbf{m m H g})\end{array}$ & $\begin{array}{c}118 \\
( \pm 8.80)\end{array}$ & $\begin{array}{c}128 \\
( \pm 18.3)\end{array}$ & $\begin{array}{c}140 \\
( \pm 16.3)\end{array}$ & 0.0002 \\
\hline HbAlc (\%) & $\begin{array}{c}8.6 \\
( \pm 2.5)\end{array}$ & $10( \pm 1.9)$ & $\begin{array}{c}9.1 \\
( \pm 1.6)\end{array}$ & 0.7063 \\
\hline \multicolumn{4}{|l|}{$\begin{array}{l}\text { Albuminuria } \\
\text { (si) }\end{array}$} & \multirow{4}{*}{0.0142} \\
\hline Normal & 12 & 0 & 1 & \\
\hline Micro & 10 & 2 & 1 & \\
\hline Macro & 4 & 4 & 5 & \\
\hline \multicolumn{4}{|l|}{$\begin{array}{l}\text { Actividad } \\
\text { física }\end{array}$} & \multirow{3}{*}{0.9132} \\
\hline Yes & 9 & 2 & 3 & \\
\hline No & 17 & 4 & 4 & \\
\hline
\end{tabular}

Fuente: elaboración propia.

Hubo una asociación significativa al comparar el tiempo desde el diagnóstico con el riesgo cardiovascular $(P=0.0037)$, mostrando un aumento en el riesgo cardiovascular a medida que la diabetes progresaba a través de los años. La presión arterial sistólica $(\mathrm{P}<0,0001)$ y los niveles de albúmina en orina $(\mathrm{P}=0,01)$ también mostraron una relación significativa con la gravedad del perfil cardiovascular.

El índice de HbA1c y la actividad física regular no tuvieron asociación estadística con el riesgo cardiovascular. 
Figura 2. Riesgo cardiovascular a 10 años

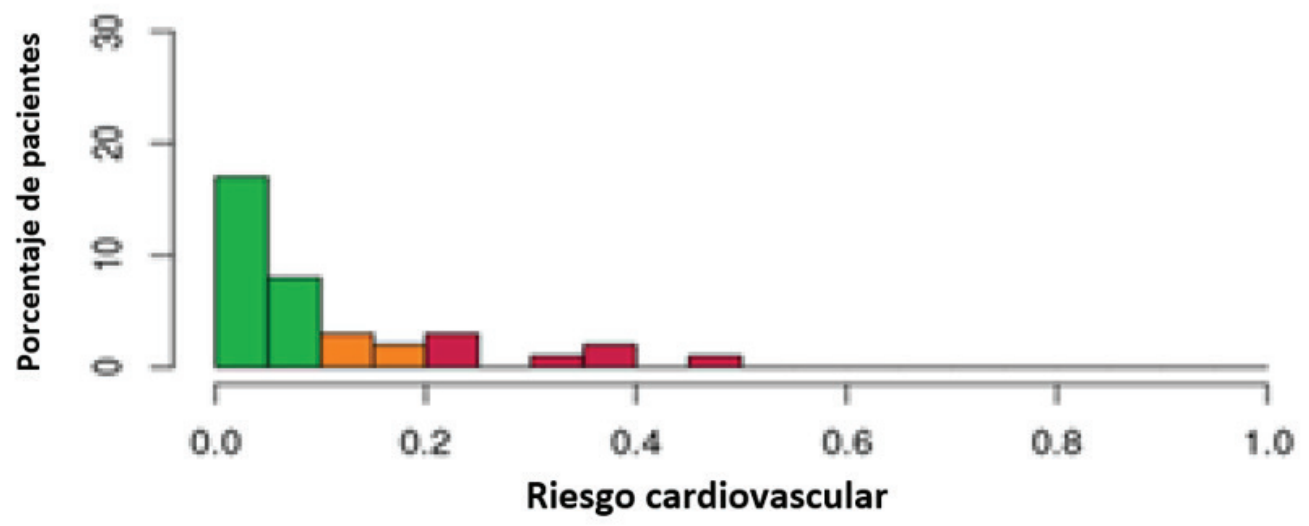

Fuente: elaboración propia.

\section{Discusión}

En general, el riesgo cardiovascular entre los participantes fue bajo. Esto podría ser secundario a que la mayoría de los pacientes en el estudio eran jóvenes, con un menor progreso de la enfermedad o con un adecuado manejo de la diabetes. Se obtuvo una relación estadísticamente significativa entre la duración de la enfermedad, presión sistólica, albuminuria y el riesgo cardiovascular, por lo que ambas hipótesis pueden ser ciertas y necesitan más estudios controlados para confirmarlo.

La duración de la diabetes ha sido demostrada como un factor de riesgo independiente en estudios previos a este estudio ${ }^{11,13}$. La presión sistólica refleja progresión del daño de la microvascular y actúa como un marcador del daño vascular en estados tardíos ${ }^{14}$; por otra parte, la albuminuria representa progresión del compromiso de la microvasculatura en estados tempranos de la enfermedad y es proporcional al riesgo cardiovascular ${ }^{11,15}$.

En cuanto a los resultados no concluyentes, el tamańo muestral y la edad promedio relativamente joven entre los participantes pudieron influenciar en los resultados. Investigaciones futuras con más participantes y mayor diversidad en la edad podrán determinar la relación entre HbA1c y la actividad física con respecto al riesgo cardiovascular en la población diabética dominicana.

La poca adherencia es un problema evidenciado de la diabetes tipo 1 , demostrándose un aumento del $28 \%$ en eficacia del manejo medido en HbA1c según mejora la adherencia ${ }^{16}$. Se propone el uso de estos datos como promotores de una atención primaria y seguimiento profesional en salud basado en evidencia hacia estos pacientes. La simpleza de estas herramientas permite el cálculo de riesgo cardiovascular en cada seguimiento con una inversión de tiempo pequeña, informando al paciente del riesgo que adquiere como medida eficaz para mejorar la adherencia ${ }^{17}$.

Entre las fortalezas de este estudio está la reducción del sesgo en la comunicación de datos, se utilizó la recolección directa y solo se incluyó aquellos resultados de laboratorio recientes y válidamente firmados. Se optó por evaluar mayormente variables objetivas, disminuyendo el sesgo de memoria. Dentro de las limitaciones del estudio se encuentran: la pobre asistencia de los pacientes luego de la programación de citas de seguimiento y la deficiente colaboración de los profesionales de salud, siento esta las causas de la disminuida cantidad de pacientes. Las investigaciones futuras deberían abordar estos problemas para obtener resultados 
más generalizables. Factores como la consistencia de los resultados con descripciones similares previas y la normalidad de distribución de datos inusual para el tamańo de muestra bajo respalda la validez del estudio.

\section{Conclusión}

El riesgo cardiovascular en la población de diabéticos tipo 1 en la población de Santiago de los Caballeros fue baja. Las variables que tuvieron la relación más cercana con el riesgo cardiovascular fueron la duración de la diabetes, la presión sanguínea sistólica y la albuminuria. El resto de las variables estudiadas no mostraron relación estadística y en futuros trabajos deben de ser estudiadas para confirmar su importancia en el riesgo cardiovascular en esta población.

Estos hallazgos representan nuevos datos considerando la ausencia de estudios relevantes en esta población. De igual forma, la calculadora de riesgos "Steno T1 Risk Engine" probó ser una herramienta útil para la evaluación del riesgo cardiovascular en estos pacientes de forma rutinaria. Se recomienda su implementación en la atención primaria de estos pacientes como medida para mejorar la adherencia al seguimiento y tratamiento. Estos hallazgos apuntan a servir como una herramienta para desarrollar más intervenciones que optimicen el control glicémico y disminuyan las complicaciones $\mathrm{CV}$ en el futuro.

\section{Referencias}

1. National Institute of Diabetes and Digestive and Kidney Diseases. "What is diabetes?”. Nov 2016. [Internet] [Citado 2018 Jul 01] Disponible en: https://www.niddk.nih.gov/health-information/ informacion-de-lasalud/diabetes

2. Cervantes-Villagrana RD, Presno-Bernal JM. Fisiopatología de la diabetes y los mecanismos de muerte de las células $\beta$ pancreáticas. Revista de Endocrinología y Nutrición. 2013;21(3):98-106.

3. World Health Organization. Informe mundial sobre la diabetes. Resumen de Orientación.
World Report on Diabetes. Executive summary; 2016.

4. Lipsky LM, Gee B, Liu A, Nansel TR. Body mass index and adiposity indicators associated with cardiovascular biomarkers in youth with type 1 diabetes followed prospectively. Pediatric obesity. 2017 Dec;12(6):468-76.

5. Evans-Cheung TC, Bodansky HJ, Parslow RC, Feltbower RG. Early deaths from ischaemic heart disease in childhood-onset type 1 diabetes. Archives of disease in childhood. 2018 Jan;24:archdischild-2017

6. Lind M, Svensson A, Kosiborod M, Gudbjörnsdottir S, Pivodic A, Wedel $\mathrm{H}$ et al. Glycemic Control and Excess Mortality in Type 1 Diabetes. New England Journal of Medicine. 2014; 371(21):1972-82.

7. Barceló A, Arredondo A, Gordillo-Tobar A, Segovia J, Qiang A. The cost of diabetes in Latin America and the Caribbean in 2015: Evidence for decision and policy makers. Journal of global health. 2017 Dec;7(2).

8. Baena-Díez J, Peñafiel J, Subirana I, Ramos R, Elosua R, Marín-Ibañez A et al. Risk of Cause-Specific Death in Individuals With Diabetes: A Competing Risks Analysis. Diabetes Care. 2016;39(11):1987-95.

9. Vistisen D, Andersen GS, Hansen CS, Hulman A, Henriksen JE, Beck-Nielsen H, Jørgensen ME. Prediction of first cardiovascular disease event in type 1 diabetes: the steno T1 risk engine. Circulation. 2016 Feb 17;CIRCULATIONAHA-115.

10. Rawshani A, Rawshani A, Franzén S, Eliasson B, Svensson AM, Miftaraj M, McGuire DK, Sattar N, Rosengren A, Gudbjörnsdottir S. Mortality and cardiovascular disease in type 1 and type 2 diabetes. New England Journal of Medicine. 2017 Apr 13;376(15):1407-18 
11. Turkbey EB, Redheuil A, Backlund JY, Small AC, Cleary PA, Lachin JM, Lima JA, Bluemke DA, Diabetes Control and Complications Trial, Epidemiology of Diabetes Interventions and Complications Research Group. Aortic distensibility in type 1 diabetes. DiabetesCare. $2013 \mathrm{Mar}$ 6:DC_120393.

12. Niedzwiecki P, Naskret D, Pilacinski S, Pempera M, Uruska A, Adamska A, Zozulinska-Ziolkiewicz D. The higher the insulin resistance the lower the cardiac output in men with type 1 diabetes during the Maximal Exercise Test. Metabolic syndrome and related disorders. 2017 Jun 1;15(5):252-7.

13. Care D. Coprogression of cardiovascular risk factors in type 1 diabetes during 30 years of follow-up in the DCCT/EDIC study. Diabetes care. 2016 Sep;39:1621-30.

14. Dost A, Bechtold-Dalla Pozza S, Bollow E, Kovacic R, Vogel P, Feldhahn L, Schwab KO,
Holl RW, Initiative DPV. Blood pressure regulation determined by ambulatory blood pressure profiles in children and adolescents with type 1 diabetes mellitus: Impact on diabetic complications. Pediatric diabetes. 2017 Dec;18(8):874-82.

15. Care D. Coprogression of cardiovascular risk factors in type 1 diabetes during 30 years of follow-up in the DCCT/EDIC study. Diabetes care. 2016 Sep;39:1621-30.

16. Hood KK, Peterson CM, Rohan JM, Drotar D. Association between adherence and glycemic control in pediatric type 1 diabetes: a metaanalysis. Pediatrics. 2009 Dec 1;124(6):e1 171-9.

17. Grover SA, Lowensteyn I, Joseph L, Kaouache M, Marchand S, Coupal L, Boudreau G. Patient knowledge of coronary risk profile improves the effectiveness of dyslipidemia therapy: the CHECK-UP study: a randomized controlled trial. Archives of Internal Medicine. $2007 \mathrm{Nov}$ 26;167(21):2296-303. 


\section{Leyenda}

Figura 1: Proceso de selección de los pacientes

Figura 2: Gráfica del riesgo cardiovascular a 10 años

Tabla 1. Descripción clínica y sociodemográfica de la muestra

Tabla 2. Descripción de variables por categoría de riesgo cardiovascular

\section{Agradecimientos}

A nuestros profesores que entregan humanidad junto a su conocimiento y a todos los diabéticos tipo 1 que luchan diariamente con su condición. A la Pontificia Universidad Madre y Maestra por promover las ciencias y su práctica dentro del marco ético.

\section{Financiamiento}

La investigación en su totalidad fue financiada por los autores. 